\title{
The Development of a Rapid Classification Scale for Sleep Quality in Community-Dwelling Older Adults - The Yilan Study, Taiwan
}

\author{
Hsi-Chung Chen (iD) ${ }^{1,2}$ \\ Nai-Wei Hsu ${ }^{3-5}$ \\ Po-Jung Pan $^{6}$ \\ Po-Hsiu Kuo (1) ${ }^{1,7}$ \\ Meng-Yueh Chien ${ }^{8}$ \\ Pesus Chou ${ }^{9}$
}

'Department of Psychiatry, National Taiwan University Hospital, Taipei,

Taiwan; ${ }^{2}$ Center of Sleep Disorders, National Taiwan University Hospital, Taipei, Taiwan; ${ }^{3}$ Division of Cardiology, Department of Internal Medicine, National Yang-Ming Chiao Tung University Hospital, Yilan, Taiwan; ${ }^{4}$ Department of Medicine, School of Medicine, National Yang-Ming Chiao Tung University, Taipei, Taiwan; ${ }^{5}$ Public Health Bureau of Yilan County, Yilan, Taiwan; ${ }^{6}$ Department of Physical Medicine and Rehabilitation \& Community Medicine Center, National Yang-Ming Chiao Tung University Hospital, Yilan, Taiwan;

${ }^{7}$ Graduate Institute of Epidemiology and Preventive Medicine, College of Public Health, National Taiwan University, Taipei, Taiwan; ${ }^{8}$ College of Medicine, National Taiwan University and the Physical Therapy Center of National Taiwan University Hospital, Taipei,

Taiwan; ${ }^{9}$ Community Medicine Research Center \& Institute of Public Health, National Yang-Ming Chiao Tung University, Taipei, Taiwan

Correspondence: Hsi-Chung Chen Department of Psychiatry, National Taiwan University Hospital, No. 7 Chung San South Road, Taipei, 10002, Taiwan

Tel +886-2-23 I 2-3456 ext. 66787

Fax +886-2-238I-320

Email hsichungchen@ntu.edu.tw
Objective: Poor sleep quality is prevailing, deleterious, but heterogeneous in older adults. This study aimed to develop a simplified instrument to screen and classify poor sleep quality in community-dwelling older adults, by which stepped care with needs-based interventions could be implemented.

Methods: Cohorts of adults aged 65 years and older were used to develop the Rapid Classification Scale for Sleep Quality (RCSSQ). Poor sleep quality was defined with the Pittsburgh Sleep Quality Index (PSQI). Established subgroups of poor sleep quality in the development dataset $(n=2622)$ were used as the criterion standard. Two independent validation datasets ( $\mathrm{n}=964$ and 193, respectively) were used to examine the external validity. Questions in the PSQI were examined by the stepwise multinomial logistic regressions to determine the optimal numbers of items in the RCSSQ. On the premise of item parsimony and instrument validity, the optimal combination of reduced items was determined.

Results: In the development dataset, the 4-item RCSSQ (RCSSQ-4) was the optimal predictive model. In terms of internal validity, the accuracy rates to identify PSQI-defined poor sleep quality and its subgroups in the developmental dataset by the RCSSQ-4 were $89.0 \%$ and $79.9 \%$, respectively. Meanwhile, the RCSSQ-4 also had good external validity in the validation datasets to detect PSQI-defined poor sleep quality (accuracy rates: 89.190.7\%). Furthermore, the profiles of PSQI component scores and comorbid conditions for the predicted subgroups in the validation dataset were comparable with the criterion standard.

Conclusion: The RCSSQ-4 is a valid instrument for screening and subgrouping poor sleep quality in community-dwelling older adults. The RCSSQ-4 may help guide tailored interventions under the context of stepped care in the community.

Keywords: older adults, screen, sleep quality, subgroup, scale, the Yilan study

\section{Introduction}

Sleep quality is imperative to health, but declines with age. ${ }^{1}$ In light of the stringency of definitions, the prevalence of poor sleep quality in community-dwelling older adults ranges between $23.2-66.9 \% .^{2-9}$ Poor sleep quality in older adults is associated with a variety of adverse health outcomes, ${ }^{2,10}$ including a higher risk for insomnia, ${ }^{11}$ depression, ${ }^{12}$ anxiety, ${ }^{5}$ falls, ${ }^{13}$ suicide,${ }^{14}$ atherosclerosis, ${ }^{15}$ declining quality of life, ${ }^{16}$ disability, ${ }^{2,6}$ cognitive impairment, ${ }^{17-19}$ and mortality. ${ }^{20}$ Furthermore, poor sleep quality in older adults is even more deleterious than insomnia without daytime repercussions. ${ }^{21,22}$ Thus, in order to promote the well-being of older adults, early 
recognition and intervention for poor sleep quality is an important issue of public health.

However, sleep quality has not been well conceptualized in the literature. ${ }^{23}$ Compared with insomnia that has clearer operational definitions, the manifestations and underlying etiology for poor sleep quality are more diverse and complex, leading to ineffective and inefficient one-size-fits-all interventions. Taking the Pittsburgh Sleep Quality Index (PSQI) as an example, this most commonly used instrument was initially designated as a uni-construct with seven underlying components. ${ }^{24}$ In recent studies, pleural constructs of the PSQI have been explored in the general, ${ }^{16,25-30}$ and older populations. ${ }^{31}$ The multi-constructs of the PSQI indicate the phenotypic heterogeneity of poor sleep quality in older adults. Under these circumstances, older adults with PSQI-defined poor sleep quality may respond differently to similar interventions. Thus, various modules of interventions are needed for the specific phenotypes of poor sleep quality. However, the different modules may demand various levels of professions, time, and labor, ${ }^{32-35}$ by which the accessibility and availability are determined. ${ }^{36}$ Accordingly, the heterogeneity of poor sleep quality not only leads to inadequate diagnosis and treatment but may also incur overtreatment or inappropriate use of hypnosedatives. ${ }^{13,37-41}$ This issue is particularly relevant in the older population, in which poor sleep quality is prevailing and who are more susceptible to the adverse impact of hypnosedatives. Empirically classifying poor sleep quality is a solution to its heterogeneity and provides an opportunity to implement personalized interventions in older adults. According to feature-based subgroups, specific modules of interventions could be designated, by which stepped care of poor sleep quality in community-dwelling older adults could be achieved. $^{42}$

Recently, according to the PSQI and the latent class analysis, the Yilan study in Taiwan has obtained empirically-derived subgroups of poor sleep quality in community-dwelling older adults. ${ }^{39}$ Three feature-based clusters have been identified and designated as "High insomnia (HI)," "Low insomnia (LI)," and "High hypnotics (HH)." The HI and LI groups were similar in the geometric profile of the component scores of the PSQI in the radar chart. They differed in the severity level of the component scores of the PSQI and comorbidity patterns. In contrast, $\mathrm{HH}$ and HI differed in the distribution of the component scores of the PSQI, in which HH had lower severity of insomnia symptoms, comparable with the LI group. In contrast, $\mathrm{HH}$ and HI shared a comparable severity of comorbidities conditions. Although these feature-based subgroups may enable the application of personalized community-based stepped care, the generalizability of the traditional clustering methods is limited due to the sample representativeness. In addition, the lengthy content of the PSQI and the cognitive demand that is needed to respond to these questions also compromises the usefulness of the PSQI for screening community-dwelling older adults. ${ }^{39}$ Thus, a simplified and valid instrument that could efficiently screen and cluster sleep quality is necessary before we can implement stepped care for poor sleep quality in community-dwelling older adults.

In summary, although poor sleep quality is prevalent and deleterious in older adults, one-size-fits-all interventions are insufficient due to the heterogeneous pattern and complex etiology of poor sleep quality. In terms of community medicine, detecting and clustering poor sleep quality with a simplified classifier could prompt need-based, stepped care of mental health. Using established subgroups in the previous Yilan study as the criterion standard, the present study aims to develop a simplified instrument to screen and subgroup the PSQI-defined poor sleep quality in community-dwelling older adults. In addition to internal validity, the generalizability of this novel instrument will be verified.

\section{Materials and Methods \\ Participants}

This study is a branch study of the umbrella project of the Yilan study in Taiwan. The Yilan study is an integrated, community-based cohort study that aims to comprehensively evaluate the mental and physical well-being of communitydwelling older adults. Older adults aged 65 years and more were randomly recruited from the Yilan city in Taiwan since 2013. The study design has been detailed elsewhere. ${ }^{43}$ The present study used two parts of the Yilan study cohort. One part (ie, the development dataset) was established in a previous study from 2013 to $2016(\mathrm{n}=2622)$, where empirically derived subgroups for the PSQI-defined poor sleep quality were identified. ${ }^{39}$ Another part of participants of the Yilan study, who had been newly recruited from 2016 to 2019 ( $\mathrm{n}=964)$, were used as the validation dataset. An additional cohort $(\mathrm{n}=193)$, which was established to investigate the PSQI-defined poor sleep quality among older adults in Taipei City (Taiwan) in $2013,{ }^{2}$ was used as an additional validation dataset to verify the external validity of the newly developed instrument. All participants in the cohort of the Yilan study were interviewed on a common set 
of variables, including sociodemographic characteristics, anthropometric data, education status, marital status, living status, lifestyles (ie, frequency of exercise per week, smoking, alcohol drinking), history of chronic medical diseases, falls, depression and anxiety, and physical disability. In contrast, only a few variables of the Yilan study were available in the Taipei City cohort because of different study designs. All interviews were conducted face-to-face by well-trained research assistants. Individuals who could not complete the interview, or were unable to cooperate because of severe physical disability or compromised cognitive function, were excluded from this study. All participants provided written informed consent, and the Institutional Review Board of the National Yang-Ming Chiao Tung University and the National Taiwan University Hospital approved this study. This study was conducted in accordance with the Helsinki Declaration of 1975, as revised in 2008.

\section{Study Design and Procedure} Subgroups Established Using the PSQI-Defined Poor Sleep Quality

This study defined poor sleep quality using the PSQI, which evaluates individuals' subjective sleep quality in the past month. The PSQI comprises 19 items and provides seven component scores, including subjective sleep quality, sleep onset latency, total sleep duration, sleep efficiency, sleep disturbances, use of sleep medication, and daytime dysfunction. ${ }^{24}$ Those who scored six points and more in the PSQI were defined as older adults with poor sleep quality.

A

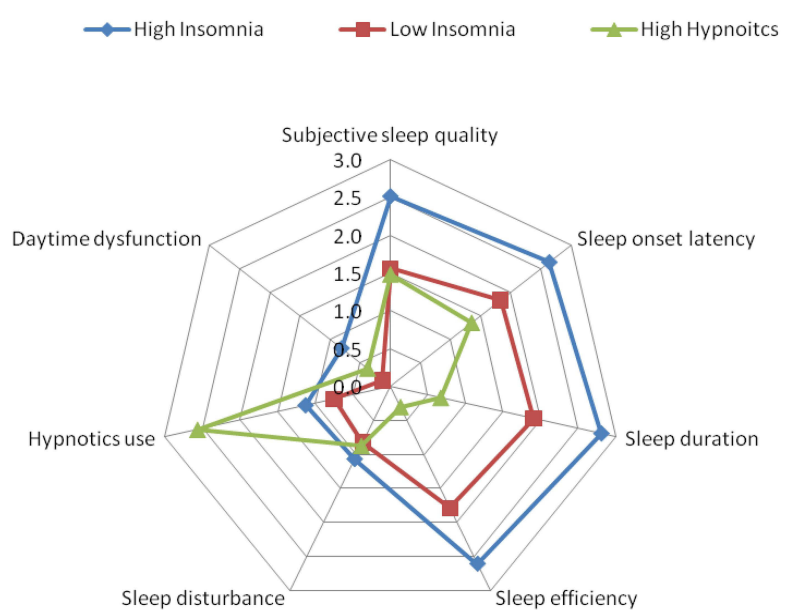

The Chinese version of the PSQI has been validated. ${ }^{44}$ In the development dataset of the Yilan study, we have used latent class analysis with seven components of the PSQI as the indicator variables to cluster those with PSQI-defined poor sleep quality (PSQI $\geq 6,38.6 \%$ ). As a result, three featurebased clusters were identified and designated as LI (21.9\%), $\mathrm{HI}(7.3 \%)$, and $\mathrm{HH}(9.4 \%)$, respectively. ${ }^{39}$ The HI group is characterized by pervasive insomnia symptoms and daytime repercussion but with a low frequency of hypnosedative use. In parallel, the LI group had a similar profile of sleep-wakerelated psychopathology with the HI but presented with a lower severity over each dimension of the PSQI component scores. In contrast, the HH group showed a comparable, lower severity across five component scores of the PSQI with LI, except for a significantly higher frequency of hypnosedative use and more prominent daytime dysfunction, similar to the HI group (Figure 1A). On the contrary, the HI and $\mathrm{HH}$ groups had a similar profile of comorbidity severity, while the LI group had better mental and physical well-being (Figure 2A). In the present study, a total of four subgroups, including the three subgroups of poor sleep quality and those with a PSQI $<6$, were defined as the criterion standard to develop and to verify the simplified screen and cluster instrument in the development dataset.

\section{Conceptualization of the Rapid Classification Scale for Sleep Quality}

On the premise of an easy application in communitydwelling older adults, by means of either paper-pencil

B
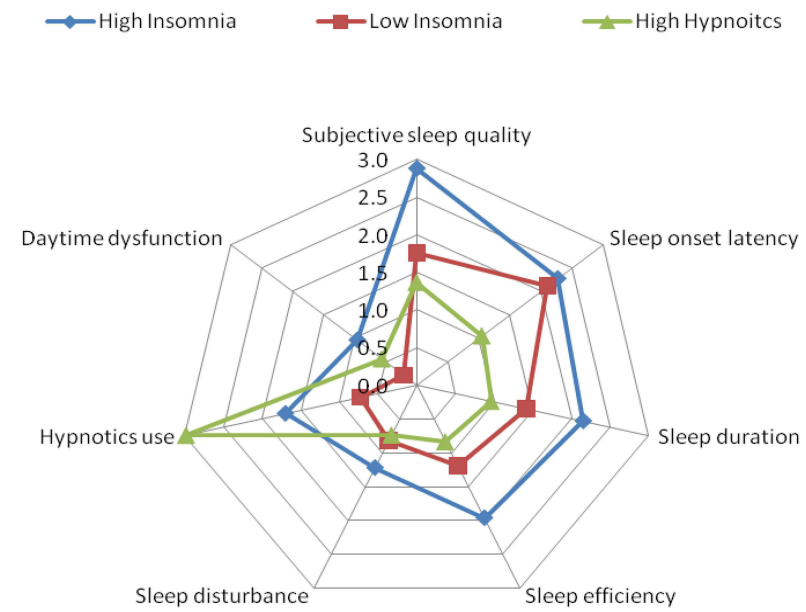

Figure I Radar charts comparing the distributions of the seven component scores of the Pittsburgh Sleep Quality Index (PSQI) between subgroups across datasets. (A) shows the original empirically-derived subgroups in the development dataset. (B) shows the three subgroups specified by the Four-item Rapid Classification Scale for Sleep Quality (RCSSQ-4) in the validation dataset. (A) is adapted from Chen HC, Hsu NW, Chou P. Subgrouping Poor Sleep Quality in Community-Dwelling Older Adults with Latent Class Analysis - The Yilan Study, Taiwan. Sci Rep. 2020;10(1):5432. ${ }^{39}$ 
A

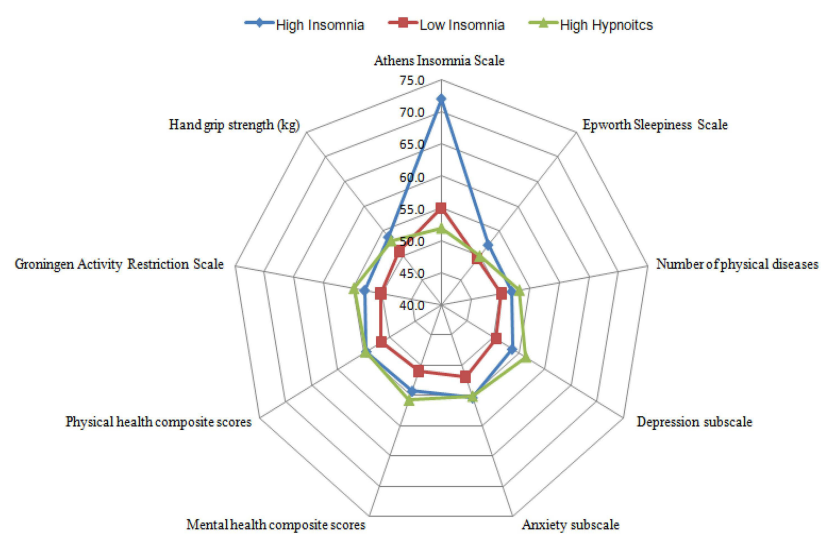

B

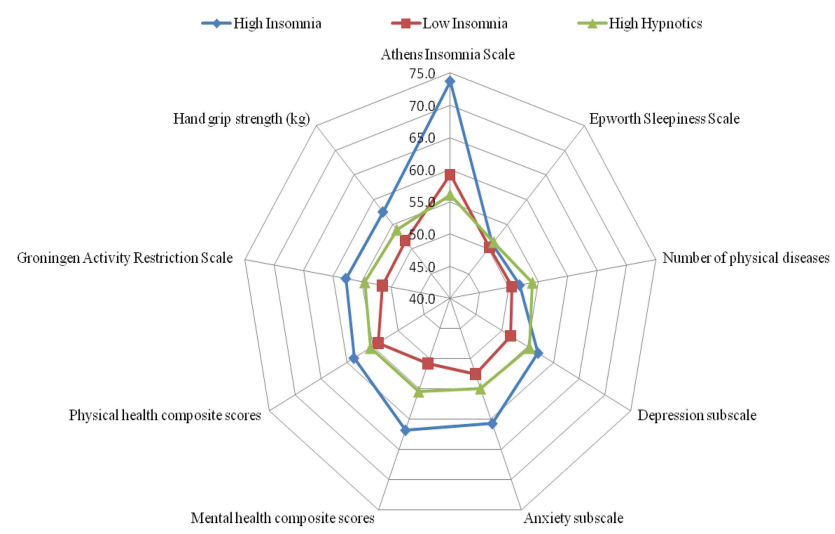

Figure 2 Radar charts comparing the clinical features between subgroups across datasets. (A) shows the original empirically-derived subgroups in the development dataset. (B) shows the three subgroups specified by the Four-item Rapid Classification Scale for Sleep Quality (RCSSQ-4) in the validation dataset. (A) is adapted from Chen HC, Hsu NW, Chou P. Subgrouping Poor Sleep Quality in Community-Dwelling Older Adults with Latent Class Analysis - The Yilan Study, Taiwan. Sci Rep. 2020;10(I):5432. ${ }^{39}$

format or the online approach, this study conceptualized and designated the screening and classifying instrument to-be as the "Rapid Classification Scale for Sleep Quality (RCSSQ)" in accordance with the following two principles:

1. Good criterion validity for PSQI-defined poor sleep quality and respective subgroups

The RCSSQ is expected to have good internal validity in the development dataset, taking the established subgroups as the criterion standard. Regarding the external validity (ie, generalizability), the RCSSQ must be able to effectively screen older adults with PSQI-defined poor sleep quality (PSQI $\geq 6$ ) and successfully classify them with a comparable feature-based difference in the validation datasets. In order to optimize the generalizability of the RCSSQ, ${ }^{45}$ in addition to the cohort in Yilan City, the present study used another cohort in Taipei City in Taiwan to examine the external validity of the RCSSQ. Compared with the agricultural, suburban Yilan City, Taipei City is a metropolitan city located in northern Taiwan, which has different sociodemographic characteristics. In the validation dataset of Yilan City, the comorbidity profile in the predictive subgroups of RCSSQ could be compared with the participants in the development dataset of Yilan City. However, because only PSQI data was available in the cohort of Taipei City, except for the examination of external validity for predicting the PSQIdefined poor sleep quality, no comorbidities pattern could be compared with the development dataset. Validity indices were illustrated with the accuracy rate, sensitivity, specificity, positive predictive value (PPV), negative predictive value (NPV), and radar charts.

2. Parsimonious items with easy-to-respond questions

In terms of community-based geriatric medicine, a simplified and easy-to-respond instrument with less cognitive demand is beneficial. Thus, in order to facilitate the convenience and intuitiveness of responding to questions, the items in the PSQI that necessitate responders to fill in actual data, such as bedtime, rise time, latency to fall asleep, and total sleep time, were not considered as candidate items for the RCSSQ. Therefore, this study selected all 13 multiple-choice items (score range: 0-3) in the PSQI as the initial set of candidate questions for the RCSSQ. All of these questions enquire about the responders' sleepwake conditions in the past four weeks, including items in the component one, "How would you rate your sleep quality overall?" (Sleep Quality); one of the items in component 2, "How often have you had trouble sleeping because you cannot get to sleep within 30 minutes?" (Sleep Latency); all eight items in component 5, "How often have you had trouble sleeping because you wake in the middle of the night or early morning?" (Sleep Disruption); "Have to get up to the bathroom?" (Nocturia); "Cannot breathe comfortably?" (Difficulty Breathing); "Cough or snore loudly?" (Disorderedbreathing Sleep); "Feel too cold?" (Cold); "Feel too hot?" (Hot); "Had bad dreams?" (Nightmares), and "Have pain?" (Pain); the item in component 6, "How 
often have you taken medicine (prescribed or "over the counter") to help you sleep?" (Hypnotics Use); and all two items in component 7, "How often have you had trouble staying awake while driving, eating meals, or engaging in social activity?" (Sleepy Activities); "How much of a problem has it been for you to keep up enough enthusiasm to get things done?" (Lacking Enthusiasm). The stepwise multinomial logistic regressions ensued to determine a most predictive set of items for the criterion standard by specifying these questions as predictors (the full model). Afterward, according to the explanatory power of each item included in the full model, a series of reduced models were specified by removing less explanatory items one-by-one. Eventually, by comparing the performance of each reduced model with the full model, a parsimonious model without compromised validity was determined as the final version of the RCSSQ.

\section{Variables Used to Profile Mental and Physical Well-Being of Predicted Subgroups}

In order to profile the mental and physical well-being with the criterion standard, this study used seven health dimensions (nine variables) to construct the radar chart, which included the number of physical morbidities, physical disability, handgrip strength, health-related quality of life (mental and physical), insomnia severity, mood (anxiety and depression), and daytime sleepiness. The definitions, contents, and instruments for measuring these healthrelated outcomes have been detailed in the previous Yilan study. ${ }^{39}$ In brief, the number of chronic physical morbidities, including hypertension, diabetes mellitus, heart disease, stroke, hyperlipidemia, cancer, and gout, was counted to reflect the disease burden. Only those who simultaneously reported to have a physical disease and to have specific treatment were coded as having a history of a specific morbidity. This approach has been proven to have good validity. ${ }^{46}$ Regarding the level of physical disability, the Groningen Activity Restriction Scale was used. Higher scores indicate a poor activity of daily life and instrumental activities of daily living. ${ }^{47}$ Handgrip strength ( $\mathrm{kg})$ was estimated using a dynamometer (Jamar, Jackson, MI, USA). The Short Form 12 Health Survey Version 2 was used to evaluate the health-related quality of life, including the mental component summary (MCS) and physical component summary (PCS). Higher scores in the component summary indicate a better health-related quality of life. ${ }^{48,49}$ The Hospital Anxiety and Depression Scale was utilized to measure the severity of depression and anxiety symptoms. ${ }^{50}$ Higher scores indicate more anxiety and depression symptoms. The severity of insomnia symptoms and daytime sleepiness were measured by the five-item Athens Insomnia Scale (AIS-5), ${ }^{51}$ and the Epworth Sleepiness Scale (ESS), ${ }^{52}$ respectively. Higher scores in the AIS-5 and the ESS indicate more severe symptoms.

\section{Statistical Analysis}

All statistical analyses were performed using SPSS for Windows, version 17.0 (SPSS Inc., Chicago, IL, USA). The univariate analyses were conducted using the $\chi^{2}$ test, $t$-test, and ANOVA. Multiple comparisons of the ANOVA were corrected using the Bonferroni method. By using four mutually exclusive subgroups of the PSQI-defined sleep quality as the dependent variables (criterion standard), forward stepwise multinomial logistic regression was used to determine the most predictive set of candidate items that were adopted from the original PSQI. This stepwise procedure determines predictors to orderly enter the final model according to the -2 log-likelihood and Chisquared test. The resulting model was regarded as the full model. Afterwards, a series of reduced models were specified by removing items in the order of least to most explainable items one-by-one. The performance of these reduced models to predict PSQI-defined poor sleep quality and respective subgroups was compared. On the premise of parsimonious items without compromised validity, the best-reduced model was selected and was designated as the final version of the RCSSQ. We also profiled radar charts to illustrate all component scores of the PSQI and mental and physical well-being of the predicted subgroups, which were clustered by the final version of the RCSSQ with the validation dataset. These sets of visualized information were compared with those of the criterion standard in the development dataset to demonstrate generalizability. For a consistent direction of comparison, all measurements of mental and physical well-being were transformed to t-scores, with higher scores indicating worse health status. A $p$-value $<0.05$ was considered statistically significant.

\section{Results}

A total of 3779 individuals in one development and two validation datasets participated in the study. Table $1 \mathrm{com}-$ pares the basic sociodemographics, lifestyle, and clinical characteristics across these three datasets. Compared with the development dataset, the participants in the validation dataset of Yilan City were younger (post-hoc $p<0.001$ ), 
Table I The Comparison of Demographic and Clinical Characteristics of Participants by Datasets*

\begin{tabular}{|c|c|c|c|c|}
\hline & \multirow{2}{*}{$\begin{array}{c}\begin{array}{c}\text { Development } \\
\text { Dataset }\end{array} \\
\text { Yilan City } \\
(n=2622)\end{array}$} & \multicolumn{2}{|c|}{ Validation Dataset } & \multirow[t]{2}{*}{$p$-value for $\chi^{2} /$ t/ANOVA Test } \\
\hline & & Yilan City $(n=964)$ & Taipei City $(n=193)$ & \\
\hline Age (years, mean $\pm S D$ ) & $76.6 \pm 6.4$ & $73.7 \pm 7.5$ & $77.8 \pm 7.3$ & $<0.001$ \\
\hline $\begin{array}{c}\text { Sex }(n, \%) \\
\text { Female }\end{array}$ & $1548(59.0)$ & $547(56.7)$ & $124(64.2)$ & 0.13 \\
\hline $\begin{array}{l}\text { Education status }(\mathrm{n}, \%) \\
\quad \text { Illiterate }\end{array}$ & $593(22.6)$ & $62(6.4)$ & - & $<0.001$ \\
\hline $\begin{array}{l}\text { Body mass index }(\mathrm{n}, \%) \\
\quad<18.5 \mathrm{~kg} / \mathrm{m}^{2} \\
18.5-23.9 \mathrm{~kg} / \mathrm{m}^{2} \\
\geq 24 \mathrm{~kg} / \mathrm{m}^{2} \\
\text { Disabled }\end{array}$ & $\begin{array}{l}105(4.0) \\
961(36.7) \\
1404(53.5) \\
152(5.8)\end{array}$ & $\begin{array}{c}35(3.6) \\
390(40.5) \\
486(50.4) \\
53(5.5)\end{array}$ & $\begin{array}{c}6(3.1) \\
82(42.5) \\
105(54.4) \\
0(0)\end{array}$ & 0.01 \\
\hline $\begin{array}{l}\text { Living status (n, \%) } \\
\text { Alone }\end{array}$ & $197(7.5)$ & $97(10.1)$ & - & 0.01 \\
\hline $\begin{array}{l}\text { Smoking status (n, \%) } \\
\text { Current smoker } \\
\text { Ex-smoker } \\
\text { Non-smoker }\end{array}$ & $\begin{array}{l}238(9.1) \\
421(16.1) \\
1963(74.9)\end{array}$ & $\begin{array}{l}75(7.8) \\
115(12.0) \\
771(80.2)\end{array}$ & - & 0.003 \\
\hline $\begin{array}{l}\text { Drinking status }(\mathrm{n}, \%) \\
\text { Current drinker } \\
\text { Ex-drinker } \\
\text { Non-drinker }\end{array}$ & $\begin{array}{c}331(12.6) \\
137(5.2) \\
2154(82.2)\end{array}$ & $\begin{aligned} 116 & (12.0) \\
55 & (5.7) \\
793 & (82.3)\end{aligned}$ & - & 0.78 \\
\hline $\begin{array}{l}\text { Frequency of exercise }(n, \%) \\
\quad<3 / \text { week }\end{array}$ & $1174(44.8)$ & $221(22.9)$ & - & $<0.001$ \\
\hline Falls in the past one year $(n, \%)$ & $454(17.3)$ & $159(16.6)$ & & 0.61 \\
\hline Pittsburgh Sleep Quality Index (mean \pm SD) & $5.1 \pm 4.0$ & $4.3 \pm 4.0$ & $6.6 \pm 4.1$ & $<0.001$ \\
\hline Athens Insomnia Scale (mean \pm SD) & $2.1 \pm 3.9$ & $1.7 \pm 3.2$ & - & 0.01 \\
\hline Epworth Sleepiness Scale (mean \pm SD) & $4.1 \pm 4.8$ & $3.0 \pm 4.2$ & $6.1 \pm 5.1$ & $<0.001$ \\
\hline Hypnotics use (days, mean \pm SD) & $6.1 \pm 11.7$ & $5.5 \pm 11.2$ & - & 0.19 \\
\hline Number of physical diseases (mean \pm SD) & $1.6 \pm 1.3$ & $1.5 \pm 1.3$ & - & 0.14 \\
\hline $\begin{array}{l}\text { Hospital Anxiety and Depression Scale } \\
\text { (mean } \pm \text { SD) } \\
\text { Depression subscale } \\
\text { Anxiety subscale }\end{array}$ & $\begin{array}{l}1.9 \pm 2.6 \\
2.3 \pm 2.9\end{array}$ & $\begin{array}{l}3.0 \pm 3.4 \\
2.7 \pm 3.1\end{array}$ & - & $\begin{array}{l}<0.001 \\
<0.001\end{array}$ \\
\hline $\begin{array}{l}\text { Short-form } 12 \text { (mean } \pm \text { SD) } \\
\text { Mental health composite scores } \\
\text { Physical health composite scores }\end{array}$ & $\begin{array}{l}58.4 \pm 7.5 \\
46.6 \pm 9.3\end{array}$ & $\begin{array}{l}57.2 \pm 7.8 \\
48.6 \pm 9.4\end{array}$ & - & $\begin{array}{l}<0.001 \\
<0.001\end{array}$ \\
\hline $\begin{array}{l}\text { Groningen Activity Restriction Scale } \\
(\text { mean } \pm \text { SD) }\end{array}$ & $23.0 \pm 11.3$ & $22.2 \pm 10.9$ & $21.6 \pm 5.4$ & 0.04 \\
\hline Hand grip strength $(\mathrm{kg}$, mean $\pm \mathrm{SD})$ & $19.5 \pm 8.5$ & $22.4 \pm 8.8$ & - & $<0.001$ \\
\hline
\end{tabular}

Note: *Some of the original variables were not available in the Taipei City cohort because the original study design differed. 
less illiterate (post-hoc $p<0.001$ ), more likely to live alone (post-hoc $p=0.01$ ), but had no significant difference for the disease burden. In terms of the sleep-wake related measurements, individuals of the Yilan City validation dataset had lower total PSQI scores (post-hoc $p<0.001$ ), AIS-5 ( $p=0.01$ ), and ESS (post-hoc $p<0.001$ ). Although the older people in the validation datasets of Yilan City had healthier physical conditions, including higher PCS $(p<0.001)$ and handgrip strength $(p<0.001)$, they had higher levels of anxiety, depression, and poor MCS (all ps $<0.001$ ). In contrast, the age (post-hoc $p=0.07$ ) and level of physical disability (post-hoc $p=0.22$ ) of participants in the validation dataset of the Taipei City had no significant difference with the development dataset. However, they had higher PSQI scores (post-hoc $p<0.001$ ) and ESS (post-hoc $p<0.001$ ), when compared with those in the development dataset.

Eight of the 13 candidate items of the PSQI that were specified using the stepwise multinomial logistic regression model to predict subgroups of the criterion standard survived (Table S1). The entry order for these eight items into this full model was (1) Sleep Quality, (2) Hypnotics Use, (3) Sleep Latency, (4) Lacking Enthusiasm, (5) Sleep Disruption, (6) Pain, (7) Sleepy Activities, and (8) Nightmares. Table 2 compares the criterion validity for PSQI-defined poor sleep quality across the full model and various reduced models. In terms of the validity for identifying PSQI $\geq 6$, at least the first three explainable items (Model V: Sleep Quality, Hypnotics Us, Sleep Onset) should be considered in order to reach $80 \%$ for sensitivity, specificity, PPV, and NPV. However, if we expect the overall accuracy rate for classifying four subgroups to reach $80 \%$, at least the five-item model (Model III: Sleep Quality, Hypnotics Use, Sleep Onset, Lacking Enthusiasm, Sleep Disruption) should be selected. Accordingly, to balance the item parsimony and criterion validity, the reduced model with three to five items was initially selected to further consider the final optimal reduced model.

Table 3 summarized the criterion validity for classifying three specific subgroups in the full model and reduced models with the three to five most predictive items. In comparison with the PPV and NPV in the full eight-item model, those of all reduced models are comparable. Regarding the sensitivity and specificity to classify the LI group, the five-item model has the best sensitivity $(63.9 \%)$, followed by the four-item model $(61.3 \%)$. The full eight-item model has the best sensitivity (64.4\%) for detecting $\mathrm{HI}$, while the three-item model has the highest sensitivity $(77.2 \%)$, followed by the four-item model $(60.6 \%)$ to identify $\mathrm{HH}$. In summary, all validity indices of the full model exceeded 50\%. Among the three-to fiveitem reduced models, on the premise that all indices of criterion validity also reach over $50.0 \%$, the four-item model of the RCSSQ (RCSSQ-4) is the most optimal option.

Detailed coefficients in the multinomial logistic regression models for each item in the full eight-item and three to five-item reduced models are summarized in Tables S1 and 4, respectively. According to these classifiers, the

Table 2 Internal Validity of the Predictive Models by Various Predictor Combinations

\begin{tabular}{|c|c|c|c|c|c|c|c|}
\hline & \multirow[t]{2}{*}{$\begin{array}{l}\text { Number of } \\
\text { Predictors }\end{array}$} & \multicolumn{5}{|c|}{$\begin{array}{l}\text { Validity for Pittsburgh Sleep Quality } \\
\qquad \text { Index } \geq 6\end{array}$} & \multirow{2}{*}{$\begin{array}{l}\text { Overall Accuracy } \\
\text { for Predicting Four } \\
\text { Subgroups (\%) }\end{array}$} \\
\hline & & $\begin{array}{c}\text { Accuracy } \\
(\%)\end{array}$ & $\begin{array}{c}\text { SEN } \\
(\%)\end{array}$ & $\begin{array}{l}\text { SPE } \\
(\%)\end{array}$ & $\begin{array}{l}\text { PPV } \\
(\%)\end{array}$ & $\begin{array}{c}\text { NPV } \\
(\%)\end{array}$ & \\
\hline Full model* & 8 & 89.9 & 83.7 & 93.9 & 89.5 & 90.1 & 81.6 \\
\hline \multicolumn{8}{|l|}{ Reduced models } \\
\hline (I). Remove Nightmare from full model & 7 & 89.7 & 82.9 & 93.9 & 89.5 & 89.7 & 81.3 \\
\hline (II). Remove Sleepy Activities from (I) & 6 & 89.4 & 82.4 & 93.9 & 89.4 & 89.5 & 81.1 \\
\hline (III). Remove Pain from (II) & 5 & 89.4 & 81.9 & 94.0 & 89.6 & 89.2 & 81.0 \\
\hline (IV). Remove Sleep Disruption from (III) & 4 & 89.0 & 83.7 & 92.3 & 87.2 & 90.0 & 79.9 \\
\hline (V). Remove Lacking Enthusiasm from (IV) & 3 & 88.7 & 82.5 & 92.7 & 87.6 & 89.4 & 78.9 \\
\hline$(\mathrm{VI})$. Remove Sleep Onset from (V) & 2 & 87.0 & 77.8 & 92.7 & 87.0 & 86.9 & 77.3 \\
\hline (VII). Remove Hypnotics Use from (VI) & I & 82.4 & 61.0 & 95.8 & 90.2 & 79.6 & 74.7 \\
\hline
\end{tabular}

Notes: *Predictors and their priority in the full model were determined by stepwise multinomial logistic regression. The full model includes predictors of sleep quality, hypnotics use, sleep onset, lacking enthusiasm, sleep disruption, pain, sleepy activities, and nightmares.

Abbreviations: SEN, sensitivity; SPE, specificity; PPV, positive predictive value; NPV, negative predictive value. 
Table 3 Internal Validity of Predictive Models for the Pittsburgh Sleep Quality Index $\geq 6$ by Various Combinations of Predictors

\begin{tabular}{|c|c|c|c|c|c|}
\hline Number of Predictors & Subgroups & SEN (\%) & SPE (\%) & PPV (\%) & NPV (\%) \\
\hline \multicolumn{6}{|l|}{ Full model } \\
\hline \multirow[t]{4}{*}{8} & \multicolumn{5}{|c|}{$\begin{array}{l}\text { Predictors: sleep quality, hypnotics use, sleep onset, lacking enthusiasm, sleep disruption, pain, sleepy activities, } \\
\text { nightmare }\end{array}$} \\
\hline & Low insomnia & 62.7 & 90.7 & 65.3 & 89.7 \\
\hline & High insomnia & 64.4 & 98.8 & 81.5 & 97.2 \\
\hline & High hypnotics & 58.9 & 95.9 & 59.7 & 95.7 \\
\hline \multicolumn{6}{|l|}{ Reduced models } \\
\hline \multirow[t]{4}{*}{5} & \multicolumn{5}{|c|}{ Predictors: sleep quality, hypnotics use, sleep onset, lacking enthusiasm, sleep disruption } \\
\hline & Low insomnia & 63.9 & 90.0 & 64.2 & 89.9 \\
\hline & High insomnia & 62.3 & 98.9 & 82.1 & 97.1 \\
\hline & High hypnotics & 49.6 & 96.4 & 59.0 & 94.9 \\
\hline \multirow[t]{4}{*}{4} & \multicolumn{5}{|c|}{ Predictors: sleep quality, hypnotics use, sleep onset, lacking enthusiasm } \\
\hline & Low insomnia & 61.3 & 89.4 & 61.8 & 89.2 \\
\hline & High insomnia & 55.5 & 99.3 & 86.2 & 96.6 \\
\hline & High hypnotics & 60.6 & 94.6 & 53.8 & 95.9 \\
\hline \multirow[t]{4}{*}{3} & \multicolumn{5}{|c|}{ Predictors: sleep quality, hypnotics use, sleep onset } \\
\hline & Low insomnia & 49.8 & 92.1 & 64.0 & 86.8 \\
\hline & High insomnia & 51.8 & 99.5 & 90.0 & 96.3 \\
\hline & High hypnotics & 77.2 & 91.4 & 48.2 & 97.5 \\
\hline
\end{tabular}

Abbreviations: SEN, sensitivity; SPE, specificity; PPV, positive predictive value; NPV, negative predictive value.

probability for each individual to be classified into specific subgroups could be estimated and compared in the validation dataset of Yilan City. As a result, each participant in the validation set could be allocated into one specific predicted subgroup. Accordingly, the external validity for the RCSSQ-4 could be evaluated. Overall, the RCSSQ-4 illustrates good generalizability to detect the PSQI-defined poor sleep quality in the validation datasets (Accuracy: $89.1-90.7 \%$; sensitivity: $87.5 \%$, and specificity: $89.7-$ 94.4\%) (Table 5).

The comparisons of the seven component scores in the PSQI and well-being conditions for the predicted subgroups obtained from the RCSSQ-4 in the validation dataset are summarized in Tables S1 and $\underline{\mathrm{S}}$. Table S2 (Yilan City) illustrates that the individuals in the predicted $\mathrm{HH}$ scored even lower than those in the predicted LI in most component scores of the PSQI, except the predicted $\mathrm{HH}$ scored higher for hypnotics use, daytime impairment and no difference with predicted LI in terms of sleep disturbance. Meanwhile, except for sleep onset latency, all the other component scores of the PSQI in the predicted HI are significantly higher than those of the predicted LI. In addition, except for hypnotics use, all other component scores in the predicted HI are higher than those in the predicted $\mathrm{HH}$. These differences are also profiled in Figure $1 \mathrm{~B}$ and are similar to those observed in the criterion standard (Figure 1A). The comparisons of the seven component scores in the PSQI for the predicted subgroups obtained from the RCSSQ-4 in the validation dataset of Taipei City are provided in Table S3.

In terms of mental and physical well-being, the predicted LI has the healthiest conditions over the entire dimensions, while the predicted $\mathrm{HI}$ and predicted $\mathrm{HH}$ have similar profiles of well-being, being poorer than that in the predicted LI. However, a few different patterns among predicted subgroups in the validation dataset (Yilan City) are observed when compared with the criterion standard (Table S4 and Figure 2). In the criterion standard, HI and $\mathrm{HH}$ showed no significant differences in anxiety symptoms and MCS (Figure 2A). However, the predicted HI shows a higher level of anxiety symptoms and poor MCS when compared with the predicted HH in the validation dataset (Figure 2B). Besides, in the criterion standard, individuals in the $\mathrm{HH}$ had significantly weaker handgrip 
Table 4 Parameter Estimates of Multinomial Logistic Regression Analyses for Predicting Subgroups of Poor Sleep Quality in the Development Dataset

\begin{tabular}{|c|c|c|c|c|c|c|}
\hline & \multicolumn{6}{|c|}{ Reference Group (Pittsburgh Sleep Quality Index $<6$ ) } \\
\hline & \multicolumn{2}{|c|}{ Low Insomnia } & \multicolumn{2}{|c|}{ High Insomnia } & \multicolumn{2}{|c|}{ High Hypnotics } \\
\hline & b (se) & OR $(95 \% \mathrm{Cl})$ & b (se) & OR $(95 \% \mathrm{Cl})$ & b (se) & OR $(95 \% \mathrm{Cl})$ \\
\hline \multicolumn{7}{|c|}{ Model I: $\mathbf{5}$ predictors } \\
\hline Intercept & $-5.20(0.23)$ & & $-16.45(0.95)$ & & $-7.35(0.37)$ & \\
\hline Sleep quality & $1.94(0.17)$ & $6.93(4.96-9.68)$ & $5.39(0.4 \mathrm{I})$ & $219.15(98.46-487.80)$ & $1.79(0.23)$ & $5.96(3.81-9.32)$ \\
\hline Hypnotics use & $0.75(0.07)$ & $2.12(1.84-2.45)$ & $0.92(0.12)$ & $2.5 \mathrm{I}(1.99-3.15)$ & $1.84(0.09)$ & $6.32(5.25-7.60)$ \\
\hline Sleep onset & $1.18(0.07)$ & $3.26(2.86-3.72)$ & $1.88(0.16)$ & $6.57(4.79-9.01)$ & $0.90(0.10)$ & $2.47(2.05-2.97)$ \\
\hline Lacking enthusiasm & $0.78(0.29)$ & $2.18(1.24-3.83)$ & $2.15(0.33)$ & $8.54(4.49-16.24)$ & $2.17(0.31)$ & $8.78(4.77-16.14)$ \\
\hline Sleep disruption & $0.68(0.08)$ & $1.98(1.70-2.30)$ & $1.09(0.14)$ & $2.98(2.28-3.89)$ & $0.48(0.11)$ & $1.62(1.31-2.00)$ \\
\hline \multicolumn{7}{|c|}{ Model II: 4 predictors } \\
\hline Intercept & $-5.21(0.23)$ & & $-16.10(0.91)$ & & $-7.36(0.37)$ & \\
\hline Sleep quality & $2.35(0.17)$ & $10.46(7.55-14.48)$ & $5.93(0.39)$ & 376.14 (175.02-808.39) & $2.06(0.22)$ & $7.86(5.07-12.19)$ \\
\hline Hypnotics use & $0.73(0.07)$ & $2.07(1.80-2.38)$ & $0.89(0.12)$ & $2.43(1.95-3.05)$ & $1.82(0.09)$ & $6.18(5.15-7.4 I)$ \\
\hline Sleep onset & $1.22(0.07)$ & $3.38(2.97-3.84)$ & $1.96(0.16)$ & 7.11 (5.17-9.77) & $0.93(0.09)$ & $2.53(2.11-3.04)$ \\
\hline Lacking enthusiasm & $0.85(0.28)$ & $2.34(1.35-4.06)$ & $2.32(0.32)$ & $10.22(5.46-19.12)$ & $2.2 I(0.3 I)$ & $9.09(4.98-16.60)$ \\
\hline \multicolumn{7}{|c|}{ Model III: 3 predictors } \\
\hline Intercept & $-5.28(0.23)$ & & $-16.01(0.89)$ & & $-7.39(0.36)$ & \\
\hline Sleep quality & $2.46(0.16)$ & II .67 (8.47-|6.09) & $6.29(0.38)$ & $54 \mid .52(256.99-|| 4 \mid .07)$ & $2.42(0.22)$ & II.26 (7.34-17.25) \\
\hline Hypnotics use & $0.72(0.07)$ & $2.06(1.79-2.36)$ & $0.86(0.11)$ & $2.36(1.90-2.93)$ & $1.76(0.08)$ & $5.82(4.89-6.91)$ \\
\hline Sleep onset & I.2I (0.07) & $3.36(2.96-3.8 I)$ & $1.90(0.16)$ & $6.72(4.92-9.17)$ & $0.93(0.09)$ & $2.5 \mathrm{I}(2.1 \mathrm{I}-3.02)$ \\
\hline
\end{tabular}

Table 5 The Validity of the Four-Item Rapid Classification Scale for Sleep Quality for the Pittsburgh Sleep Quality Index $\geq 6$

\begin{tabular}{|l|c|c|c|c|c|c|}
\hline & Prevalence of PSQI $\geq 6$ (\%) & Accuracy (\%) & SEN (\%) & SPE (\%) & PPV (\%) & NPV (\%) \\
\hline Development dataset \\
\hline \multicolumn{7}{|l|}{} \\
\hline Yilan study (n=2622) & 38.6 & 89.0 & 83.7 & 92.3 & 87.2 & 90.0 \\
\hline Validation dataset & 30.6 & 89.1 & 87.5 & 89.7 & 75.4 & 95.2 \\
\hline Yilan city (n=964) & 53.9 & 90.7 & 87.5 & 94.4 & 94.8 & 86.6 \\
Taipei city (n=193) &
\end{tabular}

Abbreviations: PSQI, the Pittsburgh Sleep Quality Index; SEN, sensitivity; SPE, specificity; PPV, positive predictive value; NPV, negative predictive value.

strength and more severe levels of physical disability than those in LI. In contrast, in the validation dataset, the predicted $\mathrm{HI}$, instead of predicted $\mathrm{HH}$, has poorer conditions than the predicted LI in these two measurements. Finally, the HI had a significantly higher level of daytime sleepiness than the LI in the development dataset, which was not observed between the predicted $\mathrm{HI}$ and predicted LI in the validation dataset. In general, the profile of mental and physical well-being in the predicted subgroups that were classified by the RCSSQ-4 with the validation dataset remained comparable with the criterion standard in the development dataset. However, regarding the withindataset comparisons, the predicted $\mathrm{HI}$ in the validation dataset had overall worse mental and physical well-being than the HI in the development dataset (Figure 2).

\section{Discussion}

The present study used large cohorts and established subgroups as the criterion standard to develop a simplified and valid instrument, the RCSSQ-4, to efficiently screen and cluster community-dwelling older adults with PSQIdefined poor sleep quality. The RCSSQ-4, which has 
balanced parsimony and validity, is an optimal option for community-based, personalized sleep care. The RCSSQ-4 has good internal and external validity to detect PSQIdefined poor sleep quality and to classify feature-based subgroups. Thus, the RCSSQ-4 is a highly applicable instrument to implement personalized care for poor sleep quality in community-dwelling older adults. Given that poor sleep quality is prevalent and deleterious but heterogeneous in older adults, our findings help to promote access to preventive medicine in sleep quality under the context of a community-based stepped care approach.

\section{Performance of the RCSSQ-4 to Screen and Classify PSQI-Defined Poor Sleep Quality}

The Validity of Screening for PSQI-Defined Poor Sleep Quality

This study demonstrated that the RCSSQ-4 had a desirable internal and external validity to identify older adults with PSQI $\geq 6$. Although the RCSSQ-4 had a very good PPV and NPV in the validation dataset of Taipei City, its superior performance in these two indices related with a higher prevalence of PSQI $\geq 6$ in Taipei City. With respect to the massive screen in the community, the outstanding sensitivity and specificity of the RCSSQ-4 are particularly noteworthy because they are independent of the prevalence rate of $\mathrm{PSQI} \geq 6$. Furthermore, we believe that, at least in the Chinese population, our findings endorse the generalizability of the RCSSQ-4, because the participants in these two validation datasets had different sociodemographic, clinical, and geographic characteristics to those in the development dataset.

\section{The Validity of Classifying Subgroups for PSQI-Defined Poor Sleep Quality}

In essence, the predicted subgroups and the criterion standard had shared patterns for the component scores of the PSQI and the well-being conditions. A few differences were observed, which may be related to the participant characteristics and the selection of candidate items for the RCSSQ. Firstly, although the individuals in the development and validation datasets of Yilan City have similar geographic characteristics, their differences in sociodemographic and clinical features may influence the resulting PSQI profile and the well-being conditions of the predicted subgroups in the validation dataset. Secondly, the most significant discrepancy when comparing the patterns of the subgroups datasets in the PSQI was that the difference between the predicted HI and predicted LI on sleep onset latency disappeared. Specifically, in the criterion standard, all component scores of the HI were higher than those of the LI. ${ }^{39}$ In the interest of the quality easy-to-respond, we removed the question about the actual sleep latency in the PSQI as a candidate item. This approach may compromise the capability of the RCSSQ to discriminate sleep latency among subgroups. Thus, the rationale and algorithm to determine the final predictive model may also influence the composition of predicted subgroups. However, despite these differences between the criterion standard and predicted subgroups, the comparable profiles of the PSQI and well-being conditions ensure the generalizability of implementing the RCSSQ-4 for personalized interventions in the community.

\section{The Balance Between Parsimony and Validity of the RCSSQ}

Based on the tradeoff between item parsimony and instrument validity, we suggest using the RCSSQ-4 for implementing community-based medicine. Among the reduced models, the three-item model with sleep quality, hypnotics use, and sleep onset enables all validity indices for detecting PSQI $\geq 6$, reaching $80 \%$ in the development dataset. However, if requesting a performance validity of $\geq 50 \%$ for subgroup classification, an additional item of Lacking Enthusiasm is necessary. In all reduced models, the models with more items had better sensitivity but poor specificity in identifying LI and HI in the development dataset. In contrast, the reduced model with fewer items is more sensitive but less specific for detecting HH. In real-world practice, the optimal version of RCSSQ can be flexibly determined, depending on the target subgroup that we intend to yield by applying the RCSSQ.

\section{Implications of the RCSSQ-4 Under the Context of Community-Based Stepped Care}

The simplified format and scoring system of the RCSSQ-4 is beneficial to conduct massive screen and clustering in the community, either by means of paper-pencil surveys or online investigations. In the viewpoint of preventive medicine, the resulting subgroups could adhere to corresponding levels of prevention. Firstly, regarding primary prevention, those designated as good sleep quality by the RCSSQ-4 may receive a general health promotion program in the community to enhance their sleep drive or 
consolidate the circadian cues. For secondary prevention, the RCSSQ-4 could detect and intervene earlier for "cases." Specifically, low professional demand but high accessibility and availability of interventions, such as selfhelp programs, online consultations, or general interventions offered by primary health providers, are indicated for the LI group, a preclinical to clinical stage of impaired sleep quality. In the HI group, in-depth diagnosis and interventions are mandatory because comorbid conditions increase. These could be provided by general physicians in primary clinics. As for the $\mathrm{HH}$ group, the indications for higher frequency and longer duration of using hypnosedatives should be carefully examined. In some patients, the exposure to hypnosedatives may be justified due to complex comorbidities and severe insomnia. In this case, the tertiary prevention should be introduced to confine the harm incurred by using hypnosedatives in older adults, such as optimizing the effectiveness of nonpharmacological interventions or a hypnotics reduction program. Obviously, the medical intervention that the HH group needs would require the high expertise that can only be provided by sleep specialists. Accordingly, using the RCSSQ-4 could help to implement preventive medicine in community-dwelling older adults under the context of personalized and stepped care.

\section{Limitations}

This study is subject to several limitations. Firstly, the RCSSQ is developed to optimize the detection and intervention for sleep quality on the population level. The RCSSQ can not replace the most commonly used PSQI, which has been the best instrument to comprehensively evaluate sleep quality in clinical settings. Secondly, although the RCSSQ-4 is sensitive to identify PSQIdefined poor sleep quality, the subgroups classified by the RCSSQ-4 may suffer from misclassification bias. It implies that most older adults with poor sleep quality can be identified by the RCSSQ-4 and can be assigned to respective modules of intervention. However, the assigned module of intervention may be imprecise because of the misclassification of the subgroups. This limitation would not affect those who are misclassified to receive modules with higher intensity, while those who are misclassified to modules with lower intensity could be referred to a higher level of stepped care if the treatment effect is limited. Thirdly, although validation datasets were used to examine the external validity of the RCSSQ-4, the inherent limitation of within-sample analyses of the traditional clustering methods makes it difficult to have adequate representativeness across different ages, ethnicities, and cultures. Fourthly, although the subgroups that are defined in this study suggest different interventions, the cross-sectional design of this study did not allow us to make causal inferences for the relationship between poor sleep quality and co-occurring mental or physical conditions. Thus, the suggested modules of interventions for each subgroup in this study are merely based on clinical experience. Finally, to enhance the feasibility of implementing the RCSSQ for older adults in the community, as an instrument for mass screening and subgrouping, we used the PSQI and its items to define sleep quality, classify subgroups, and develop predictive models in the present study. The PSQI has been commonly used for convergent validity, suggesting that it can be considered as an accepted reference for self-perceived sleep quality. ${ }^{53}$ However, sleep quality represents a complex construct that comprises several aspects of the sleep phenomenon and opposes a simple definition. ${ }^{54}$ Applying different measurements or definitions for sleep quality, such as other subjective instruments and objective parameters of sleep, may yield different subgroups and predictive models.

\section{Conclusion}

For the community medicine of the aging society, the RCSSQ-4 could serve as a rapid and efficient instrument to screen and classify poor sleep quality in older adults. By applying the RCSSQ-4, we may streamline the cost of various adverse outcomes related to poor sleep quality from the perspective of preventive medicine. Besides, it also becomes possible to deliver personalized interventions and achieve stepped care at the community level. In the future, longitudinal studies would be required to examine the validity of subgroups by comparing differences in clinical courses, health outcomes, treatment effects, and biological markers. In addition, novel clustering approaches, such as machine learning, ${ }^{55}$ might help to refine the validity of RCSSQ and promote generalizability. Hopefully, the implementation of the RCSSQ-4 will help guide need-based interventions, which will eventually lead to community-level precision medicine.

\section{Abbreviations}

AIS-5, the 5-item Athens Insomnia Scale; ESS, Epworth Sleepiness Scale; HI, high insomnia group; HH, high hypnotics group; LI, low insomnia group; MCS, mental component summary; NPV, negative predictive value; 
PCS, physical component summary; PPV, positive predictive value; PSQI, Pittsburgh Sleep Quality Index; RCSSQ, Rapid Classification Scale for Sleep Quality; RCSSQ-4, the 4-item Rapid Classification Scale for Sleep Quality.

\section{Data Sharing Statement}

The datasets generated during and/or analysed during the current study are available from the corresponding author on reasonable request.

\section{Acknowledgments}

The authors acknowledge statistical assistance provided by the Center of Statistical Consultation and Research in the Department of Medical Research, National Taiwan University Hospital. The authors would also like to thank the Yang-Ming Crusaders, Mr. Da-Wei Lin, Ms. Yu-Hui Lin, Mr. Chia-Hsiang Lin, and Ms. Tzu-Chun Lo for their help with data collection.

\section{Author Contributions}

All authors contributed to data analysis, drafting or revising the article, have agreed on the journal to which the article will be submitted, gave final approval of the version to be published, and agree to be accountable for all aspects of the work.

\section{Funding}

This work was supported by the Ministry of Science and Technology, Taiwan [MOST-107-2314-B-010 -049], [MOST-108-2314-B-002 -110 -MY2], and [MOST-1102314-B-002 -096 -MY3].

\section{Disclosure}

We have no conflict of interest to declare.

\section{References}

1. Ancoli-Israel S. Sleep and its disorders in aging populations. Sleep Med. 2009;10(Suppl 1):S7-S11. doi:10.1016/j.sleep.2009.07.004

2. Chien MY, Chen HC. Poor sleep quality is independently associated with physical disability in older adults. J Clin Sleep Med. 2015;11 (3):225-232. doi:10.5664/jcsm.4532

3. Chen HC, Hsu NW, Chou P. The association between sleep duration and hand grip strength in community-dwelling older adults: the Yilan Study, Taiwan. Sleep. 2017;40(4):ZSX021. doi:10.1093/sleep/zsx021

4. Wu CY, Su TP, Fang CL, Yeh Chang M. Sleep quality among community-dwelling elderly people and its demographic, mental, and physical correlates. $J$ Chin Med Assoc. 2012;75(2):75-80. doi:10.1016/j.jcma.2011.12.011

5. Luo J, Zhu G, Zhao Q, et al. Prevalence and risk factors of poor sleep quality among Chinese elderly in an urban community: results from the Shanghai aging study. PLoS One. 2013;8(11):e81261. doi:10.1371/ journal.pone.0081261
6. Beaudreau SA, Spira AP, Stewart A, et al. Validation of the Pittsburgh Sleep Quality Index and the Epworth Sleepiness Scale in older black and white women. Sleep Med. 2012;13(1):36-42. doi:10.1016/j.sleep.2011.04.005

7. Chang KJ, Son SJ, Lee Y, et al. Perceived sleep quality is associated with depression in a Korean elderly population. Arch Gerontol Geriatr. 2014;59(2):468-473. doi:10.1016/j.archger.2014.04.007

8. McHugh JE, Casey AM, Lawlor BA. Psychosocial correlates of aspects of sleep quality in community-dwelling Irish older adults. Aging Ment Health. 2011;15(6):749-755. doi:10.1080/ 13607863.2011.562180

9. Wang P, Song L, Wang K, et al. Prevalence and associated factors of poor sleep quality among Chinese older adults living in a rural area: a population-based study. Aging Clin Exp Res. 2019;32(1):125-231.

10. Del Brutto OH, Mera RM, Sedler MJ, et al. The effect of age in the association between frailty and poor sleep quality: a population-based study in community-dwellers (The Atahualpa Project). $J$ Am Med Dir Assoc. 2016;17(3):269-271. doi:10.1016/j.jamda.2015.12.009

11. Morin CM, Belleville G, Belanger L, Ivers $\mathrm{H}$. The insomnia severity index: psychometric indicators to detect insomnia cases and evaluate treatment response. Sleep. 2011;34(5):601-608. doi:10.1093/sleep/ 34.5.601

12. Grandner MA, Kripke DF, Yoon I-Y, Youngstedt SD. Criterion validity of the Pittsburgh Sleep Quality Index: investigation in a non-clinical sample. Sleep Biol Rhythms. 2006;4(2):129-139. doi:10.1111/j.1479-8425.2006.00207.x

13. Min Y, Kirkwood CK, Mays DP, Slattum PW. The effect of sleep medication use and poor sleep quality on risk of falls in community-dwelling older adults in the US: a prospective cohort study. Drugs Aging. 2016;33(2):151-158. doi:10.1007/s40266-0150339-9

14. Bernert RA, Joiner TE Jr, Cukrowicz KC, Schmidt NB, Krakow B. Suicidality and sleep disturbances. Sleep. 2005;28(9):1135-1141. doi:10.1093/sleep/28.9.1135

15. Del Brutto OH, Mera RM, Penaherrera E, Costa AF, Penaherrera R, Castillo PR. On the association between sleep quality and arterial stiffness: a population study in community-dwelling older adults living in rural ecuador (The Atahualpa Project). J Clin Sleep Med. 2019;15(8):1101-1106. doi:10.5664/jcsm.7798

16. Nicassio PM, Ormseth SR, Custodio MK, Olmstead R, Weisman MH, Irwin MR. Confirmatory factor analysis of the Pittsburgh Sleep Quality Index in rheumatoid arthritis patients. Behav Sleep Med. 2014;12 (1):1-12. doi:10.1080/15402002.2012.720315

17. Miyata S, Noda A, Iwamoto K, Kawano N, Okuda M, Ozaki N. Poor sleep quality impairs cognitive performance in older adults. J Sleep Res. 2013;22(5):535-541. doi:10.1111/jsr.12054

18. Nakakubo S, Makizako H, Doi T, et al. Impact of poor sleep quality and physical inactivity on cognitive function in community-dwelling older adults. Geriatr Gerontol Int. 2017;17(11):1823-1828. doi:10.1111/ggi.12973

19. Potvin O, Lorrain D, Forget H, et al. Sleep quality and 1-year incident cognitive impairment in community-dwelling older adults. Sleep. 2012;35(4):491-499. doi:10.5665/sleep. 1732

20. Martin JL, Fiorentino L, Jouldjian S, Mitchell M, Josephson KR, Alessi CA. Poor self-reported sleep quality predicts mortality within one year of inpatient post-acute rehabilitation among older adults. Sleep. 2011;34(12):1715-1721. doi:10.5665/sleep.1444

21. Chiu HF, Leung T, Lam LC, et al. Sleep problems in Chinese elderly in Hong Kong. Sleep. 1999;22(6):717-726. doi:10.1093/sleep/ 22.6.717

22. Ohayon MM. Epidemiology of insomnia: what we know and what we still need to learn. Sleep Med Rev. 2002;6(2):97-111. doi:10.1053/ smrv.2002.0186

23. Ohayon M, Wickwire EM, Hirshkowitz M, et al. National sleep foundation's sleep quality recommendations: first report. Sleep Health. 2017;3(1):6-19. doi:10.1016/j.sleh.2016.11.006 
24. Buysse DJ, Reynolds CF 3rd, Monk TH, Berman SR, Kupfer DJ. The Pittsburgh Sleep Quality Index: a new instrument for psychiatric practice and research. Psychiatry Res. 1989;28(2):193-213. doi:10.1016/0165-1781(89)90047-4

25. Babson KA, Blonigen DM, Boden MT, Drescher KD, Bonn-Miller MO. Sleep quality among U.S. military veterans with PTSD: a factor analysis and structural model of symptoms. J Trauma Stress. 2012;25 (6):665-674. doi:10.1002/jts. 21757

26. Casement MD, Harrington KM, Miller MW, Resick PA. Associations between Pittsburgh Sleep Quality Index factors and health outcomes in women with posttraumatic stress disorder. Sleep Med. 2012;13 (6):752-758. doi:10.1016/j.sleep.2012.02.014

27. Magee CA, Caputi P, Iverson DC, Huang X-F. An investigation of the dimensionality of the Pittsburgh Sleep Quality Index in Australian adults. Sleep Biol Rhythms. 2008;6(4):222-227. doi:10.1111/j.1479-8425.2008.00371.x

28. Otte JL, Rand KL, Carpenter JS, Russell KM, Champion VL. Factor analysis of the Pittsburgh Sleep Quality Index in breast cancer survivors. J Pain Symptom Manage. 2013;45(3):620-627. doi:10.1016/j.jpainsymman.2012.03.008

29. Tomfohr LM, Schweizer CA, Dimsdale JE, Loredo JS. Psychometric characteristics of the Pittsburgh Sleep Quality Index in English speaking non-Hispanic whites and English and Spanish speaking Hispanics of Mexican descent. J Clin Sleep Med. 2013;9(1):61-66. doi: $10.5664 / \mathrm{jcsm} .2342$

30. Mariman A, Vogelaers D, Hanoulle I, Delesie L, Tobback E, Pevernagie D. Validation of the three-factor model of the PSQI in a large sample of chronic fatigue syndrome (CFS) patients. J Psychosom Res. 2012;72(2):111-113. doi:10.1016/j.jpsychores.2011.11.004

31. Cole JC, Motivala SJ, Buysse DJ, Oxman MN, Levin MJ, Irwin MR. Validation of a 3-factor scoring model for the Pittsburgh sleep quality index in older adults. Sleep. 2006;29(1):112-116. doi:10.1093/sleep/ 29.1.112

32. Black DS, O'Reilly GA, Olmstead R, Breen EC, Irwin MR. Mindfulness meditation and improvement in sleep quality and daytime impairment among older adults with sleep disturbances: a randomized clinical trial. JAMA Intern Med. 2015;175 (4):494-501. doi:10.1001/jamainternmed.2014.8081

33. Campanini MZ, Guallar-Castillon P, Rodriguez-Artalejo F, LopezGarcia E. Mediterranean diet and changes in sleep duration and indicators of sleep quality in older adults. Sleep. 2017;40(3). doi:10.1093/sleep/zsw083

34. Chan AW, Yu DS, Choi KC, Lee DT, Sit JW, Chan HY. Tai chi qigong as a means to improve night-time sleep quality among older adults with cognitive impairment: a pilot randomized controlled trial. Clin Interv Aging. 2016;11:1277-1286. doi:10.2147/CIA.S111927

35. Seol J, Abe T, Fujii Y, Joho K, Okura T. Effects of sedentary behavior and physical activity on sleep quality in older people: a cross-sectional study. Nurs Health Sci. 2019;22(1):64-71.

36. Espie CA. "Stepped care": a health technology solution for delivering cognitive behavioral therapy as a first line insomnia treatment. Sleep. 2009;32(12):1549-1558. doi:10.1093/sleep/32.12.1549

37. Edinger JD, Buysse DJ, Deriy L, et al. Quality measures for the care of patients with insomnia. J Clin Sleep Med. 2015;11(3):311-334. doi: $10.5664 / \mathrm{jcsm} .4552$

38. Sateia MJ, Buysse DJ, Krystal AD, Neubauer DN. Adverse effects of hypnotic medications. J Clin Sleep Med. 2017;13(6):839.

39. Chen HC, Hsu NW, Chou P. Subgrouping poor sleep quality in community-dwelling older adults with latent class analysis - the Yilan Study, Taiwan. Sci Rep. 2020;10(1):5432. doi:10.1038/ s41598-020-62374-4
40. Buscemi N, Vandermeer B, Friesen C, et al. The efficacy and safety of drug treatments for chronic insomnia in adults: a meta-analysis of RCTs. J Gen Intern Med. 2007;22(9):1335-1350. doi:10.1007/ s11606-007-0251-z

41. Chen SY, Chen CH, Lo C, et al. Differential co-occurring patterns between depressive symptomatology and sleep-wake-related disturbances in mood disorders. Nat Sci Sleep. 2021;13:503-514. doi:10.2147/NSS.S301357

42. O’Donohue WT, Draper C; SpringerLink (Online service). Stepped Care and e-Health Practical Applications to Behavioral Disorders. New York, NY: Springer Science+Business Media, LLC; 2011. doi:10.1007/978-1-4419-6510-3

43. Hsu NW, Tsao HM, Chen HC, Chou P. Anxiety and depression mediate the health-related quality of life differently in patients with cardiovascular disease and stroke-preliminary report of the Yilan study: a population-based community health survey. PLoS One. 2014;9(9):e107609. doi:10.1371/journal.pone.0107609

44. Tsai PS, Wang SY, Wang MY, et al. Psychometric evaluation of the Chinese version of the Pittsburgh Sleep Quality Index (CPSQI) in primary insomnia and control subjects. Qual Life Res. 2005;14 (8):1943-1952. doi:10.1007/s11136-005-4346-x

45. Ferguson L. External validity, generalizability, and knowledge utilization. J Nurs Scholarsh. 2004;36(1):16-22. doi:10.1111/j.15475069.2004.04006.x

46. Wu CS, Lai MS, Gau SS, Wang SC, Tsai HJ. Concordance between patient self-reports and claims data on clinical diagnoses, medication use, and health system utilization in Taiwan. PLoS One. 2014;9(12): e112257. doi:10.1371/journal.pone.0112257

47. Suurmeijer TP, Doeglas DM, Moum T, et al. The Groningen activity restriction Scale for measuring disability: its utility in international comparisons. Am J Public Health. 1994;84(8):1270-1273. doi:10.2105/AJPH.84.8.1270

48. Bullinger M, Alonso J, Apolone G, et al. Translating health status questionnaires and evaluating their quality: the IQOLA project approach. International Quality of Life Assessment. $J$ Clin Epidemiol. 1998;51(11):913-923. doi:10.1016/S0895-4356(98) 00082-1

49. Tseng HM, Lu JF, Gandek B. Cultural issues in using the SF-36 health survey in Asia: results from Taiwan. Health Qual Life Outcomes. 2003;1(1):72. doi:10.1186/1477-7525-1-72

50. Zigmond AS, Snaith RP. The hospital anxiety and depression scale. Acta Psychiatr Scand. 1983;67(6):361-370. doi:10.1111/j.16000447.1983.tb09716.x

51. Chiang $\mathrm{HL}$, Chen $\mathrm{HC}, \mathrm{Bai} \mathrm{CH}$, et al. A validation study of the Chinese version of the Athens Insomnia Scale. Taiwan J Psychiatry. 2009;23(1):43-52.

52. Johns MW. A new method for measuring daytime sleepiness: the Epworth sleepiness Scale. Sleep. 1991;14(6):540-545. doi:10.1093/ sleep/14.6.540

53. Fabbri M, Beracci A, Martoni M, Meneo D, Tonetti L, Natale V. Measuring subjective sleep quality: a review. Int $J$ Environ Res Public Health. 2021;18(3):1082. doi:10.3390/ijerph18031082

54. Landry GJ, Best JR, Liu-Ambrose T. Measuring sleep quality in older adults: a comparison using subjective and objective methods. Front Aging Neurosci. 2015;7:166. doi:10.3389/fnagi.2015.00166

55. Blanken TF, Benjamins JS, Borsboom D, et al. Insomnia disorder subtypes derived from life history and traits of affect and personality. Lancet Psychiatry. 2019;6(2):151-163. doi:10.1016/S2215-0366(18) 30464-4 


\section{Publish your work in this journal}

Nature and Science of Sleep is an international, peer-reviewed, open access journal covering all aspects of sleep science and sleep medicine, including the neurophysiology and functions of sleep, the genetics of sleep, sleep and society, biological rhythms, dreaming, sleep disorders and therapy, and strategies to optimize healthy sleep.
The manuscript management system is completely online and includes a very quick and fair peer-review system, which is all easy to use. Visit http://www.dovepress.com/testimonials.php to read real quotes from published authors. 